\title{
Summary of the working group on Higgs physics and physics beyond the Standard Model
}

\author{
Chris Hays $^{1}$, Roman Kogler ${ }^{2 *}$, and Eleni Vryonidou ${ }^{3}$ \\ ${ }^{1}$ Oxford University, ${ }^{2}$ University of Hamburg, ${ }^{3}$ NIKHEF \\ E-mail: chris.hays@physics.ox.ac.uk, roman.kogler@uni-hamburg.de, \\ evryonidouegmail.com
}

\begin{abstract}
We review the contributions of the Higgs and beyond-the-Standard-Model working group, covering Higgs boson measurements, Higgs boson searches as a probe of physics beyond the Standard Model, other new particles and forces, dark matter, and supersymmetry. We focus on recent measurements with $\sqrt{s}=13 \mathrm{TeV} p p$ collision data.
\end{abstract}

XXV International Workshop on Deep-Inelastic Scattering and Related Subjects 3-7 April 2017

University of Birmingham, $U K$

\footnotetext{
* Speaker.
} 


\section{Introduction}

Experiments at the Large Hadron Collider now have larger datasets from $\sqrt{s}=13 \mathrm{TeV} p p$ collisions (Run 2) than from the $\sqrt{s}=7$ and $8 \mathrm{TeV}$ run (Run 1). At this higher energy the cross sections of SM processes increase by a factor of 2-4, and those of high-scale new physics processes increase by 1-3 orders of magnitude. Measurements of the Higgs boson have demonstrated the expected increase in production cross section, while searches for rare Higgs processes are now starting to see evidence of these processes. Other Higgs-related searches have probed non-SM couplings and extended Higgs sectors. Resonance searches are sensitive to new particles at unprecedented masses, dark matter searches are expanding to untested processes, and supersymmetry searches continue to reduce the available parameter space. We review the experimental and theoretical progress in these areas presented in the Higgs and beyond-the-Standard-Model working group at the DIS conference, with an emphasis on results with the full 2015-2016 LHC data set.

\section{Higgs boson measurements and searches}

The Higgs boson was discovered in its decay to dibosons and these decay channels are providing the first Higgs measurements of Run 2. Higgs boson decays to fermions have not been observed by a single experiment, though there is evidence for these decays and for Higgs interactions with the top quark through $t t H$ production. Searches for rarer decays are becoming more sensitive, as are searches for additional potential Higgs bosons.

Both ATLAS and CMS have observed $H \rightarrow Z Z^{*} \rightarrow 4 \ell$ decays in Run 2. ATLAS has performed a cross section measurement [1] $\left(\sigma_{\mathrm{tot}}=81_{-16}^{+18} \mathrm{pb}\right.$, compatible with the SM predicted total Higgs boson production cross section of $55.5_{-4.4}^{+3.8} \mathrm{pb}$ ) and CMS has directly constrained the width of the Higgs boson to be less than $3.9 \mathrm{GeV}$ at $95 \%$ confidence level (C.L.) [2]. CMS has also measured events at higher invariant mass and used the ratio with respect to the resonance cross section to obtain an indirect upper bound of $41 \mathrm{MeV}$ on the width of the Higgs boson in the absence of new particles.

With the higher event yields in $H \rightarrow \gamma \gamma$ decays, ATLAS has measured the separate cross sections for gluon-fusion $(\mathrm{ggH})$ and vector-boson-fusion (VBF) production in a fit that also includes the $t t H$ and $V H$ production processes [3]. The results, $\sigma_{\mathrm{ggH}} \times \mathscr{B}(H \rightarrow \gamma \gamma)=63_{-29}^{+30} \mathrm{fb}$ and $\sigma_{\mathrm{VBF}} \times$ $\mathscr{B}(H \rightarrow \gamma \gamma)=17.8_{-5.7}^{+6.3} \mathrm{fb}$ deviate from the SM predictions by a little more than $1 \sigma\left(\sigma_{\mathrm{ggH}} / \sigma_{\mathrm{SM}}=\right.$ 0.59 and $\left.\sigma_{\mathrm{VBF}} / \sigma_{\mathrm{SM}}=2.24\right)$. CMS has performed a differential cross section measurement in this channel [4], measuring the Higgs boson $p_{T}$ to beyond $350 \mathrm{GeV}$ (Figure 1 left). At this momentum it is important for the $\mathrm{ggH}$ predictions to include the finite top-quark mass, since it reduces the cross section by more than 30\% [5] (Figure 1 right).

Measurements of the Higgs-boson couplings to fermions are making substantial progress. An ATLAS fit for the $V H$ cross section with $H \rightarrow b b$ has an uncertainty that is $50 \%$ of the SM prediction, and constrains the cross section to be less than 1.2 times the SM prediction at 95\% C.L [6]. An ATLAS search for the rare decay $H \rightarrow \mu \mu$ yields an upper bound on the cross section that is 3 times the SM prediction [7].

The experiments are rapidly approaching observation of $t t H$ production. ATLAS has exceeded its Run 1 sensitivity with half the Run 2 data [8], and CMS has a $3.3 \sigma$ excess of $t t H$ candidate 

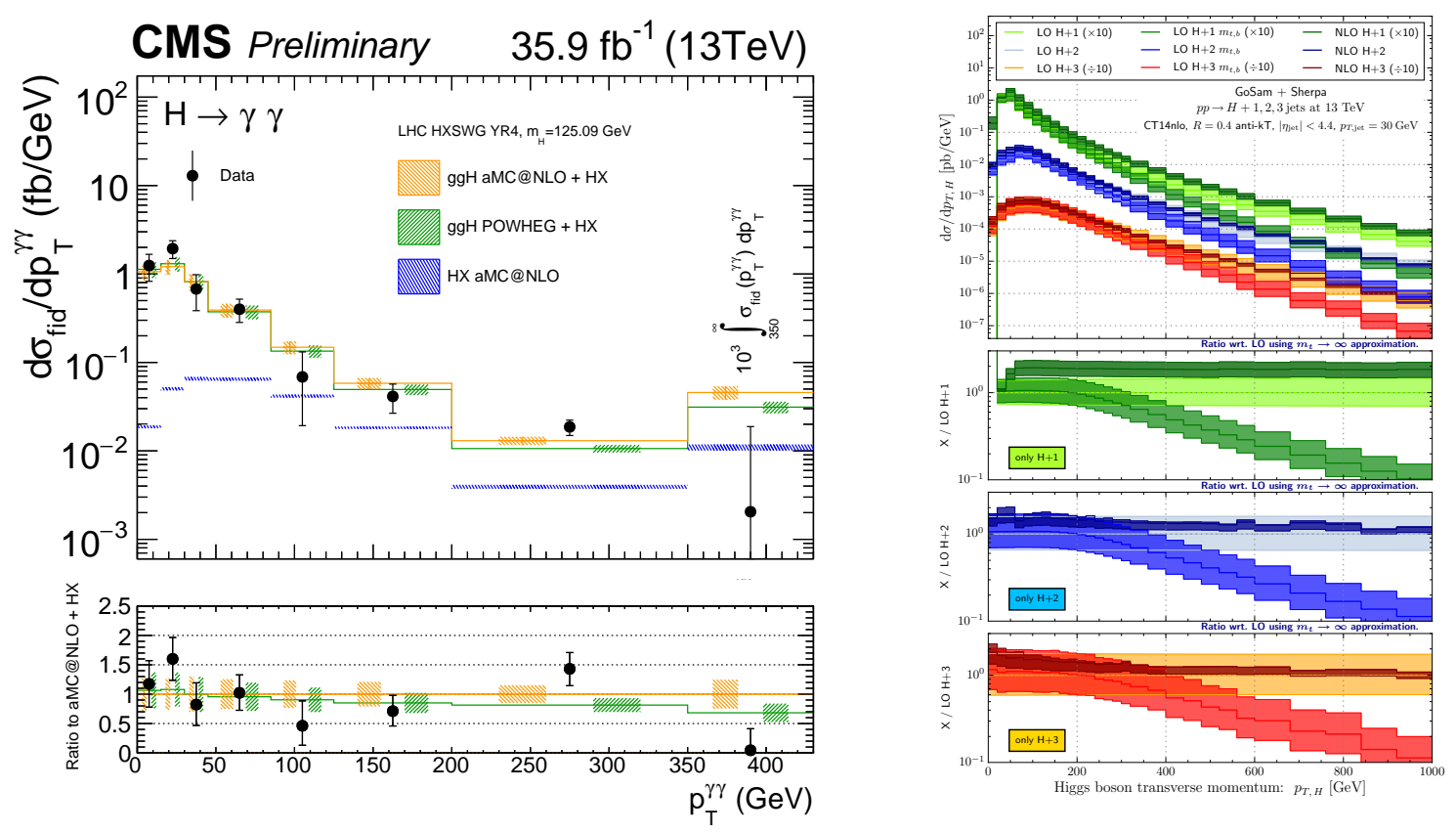

Figure 1: Left: The $p_{T}$ of the Higgs boson measured by CMS in $H \rightarrow \gamma \gamma$ decays [4]. Right: The $p_{T}$ of the Higgs boson for different parton multiplicities, and the impact of using the finite top-quark mass in the calculation [5].

events above the background using only Higgs-boson decays to $W W, Z Z$, or $\tau \tau$ that result in two or three charged leptons ( $e$ or $\mu$ ) [9]. The modelling of the signal has been improved with nextto-next-to-leading logarithm resummation [10], while the important $t t b b$ background now has a PowheG+OPEnLoops Monte Carlo implementation that includes effect of the non-zero $b$-quark mass [11].

Thus far the measurements of the Higgs boson are consistent with the SM predictions. A general characterization of non-SM effects can be obtained in the context of an effective field theory (EFT). The leading operators are those at dimension 6, which capture effects of both rate and distribution. A recent implementation in MadGraph includes corrections at NLO in QCD for $V H$ and VBF [12], and the impact of EFT operators on angular distributions in $H \rightarrow Z Z^{*} \rightarrow 4 \ell$ decays has recently been studied [13]. Tests for new physics in decay rates rely on the calculation of the total width, which has been shown to converge well in a calculation of the $\alpha_{s}^{4}$ contributions to the decays $H \rightarrow b b$ and $H \rightarrow g g$ [14].

The couplings in the Higgs sector span a wide range of values and there are many rare processes resulting from the subleading couplings. One of the most important processes is di-Higgs production, which depends on the Higgs-boson self-coupling. Many decays channels are used to search for pairs of Higgs bosons produced via gluon fusion, and rely on accurate modelling of the production kinematics. The recent inclusion of the finite top-quark mass in Monte Carlo predictions of di-Higgs production provides a more accurate model of the di-Higgs $p_{T}$, as shown in Figure 2 (left). Sensitivity to SM di-Higgs production will only be possible at the HL-LHC and 

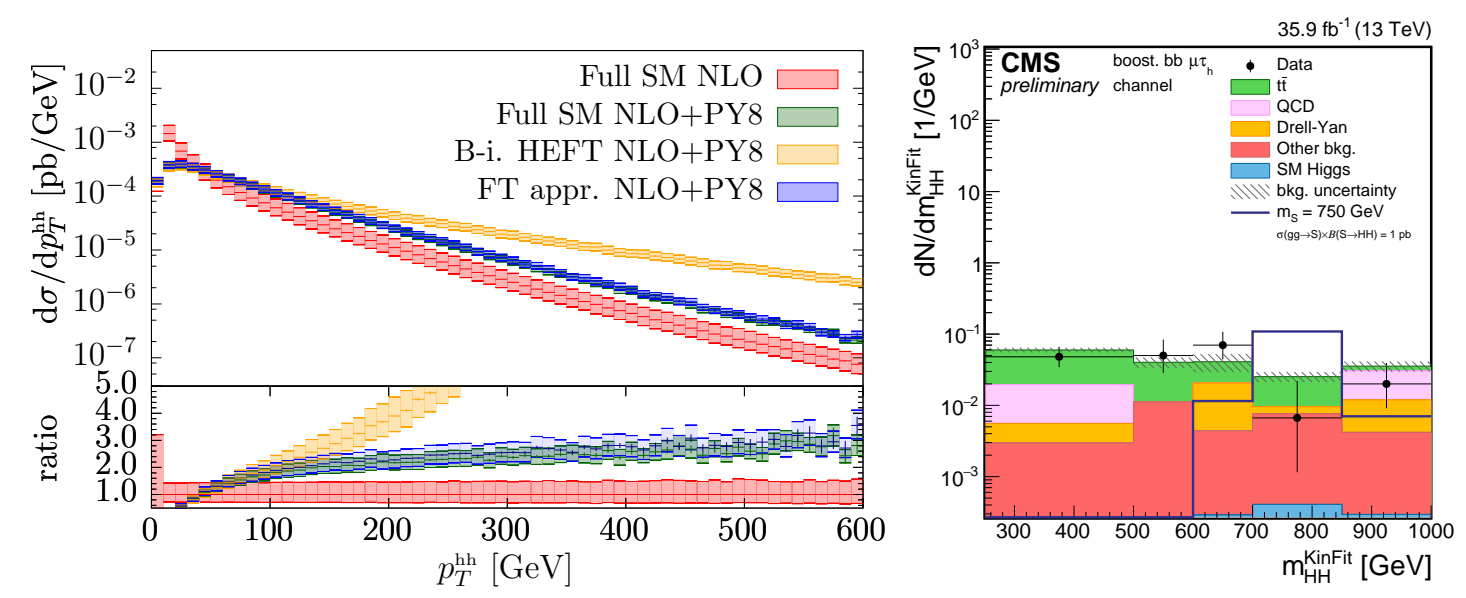

Figure 2: Left: The $p_{T}$ of the Higgs-boson pair in di-Higgs production for various calculations [15]. Right: The invariant mass of the candidate Higgs-boson pair in a CMS search for resonant production of a scalar decaying to $H H \rightarrow b b \tau \tau[17]$.

will require the inclusion of many channels and multiple production mechanisms. However, other production mechanisms are not necessarily most sensitive to the Higgs self-coupling. A study of di-Higgs production in vector-boson fusion at the HL-LHC and a future circular collider (FCC) suggests that this process can best be used to constrain the $V V H H$ coupling rather than the Higgs boson self-coupling [16]. Current searches for di-Higgs production focus on gluon-fusion production and generally interpret the results in the context of new resonances. The distribution of the di-Higgs mass in a CMS search for $H H \rightarrow b b \tau \tau$ is shown in Figure 2 (right) [17].

Other small couplings can be constrained by searching for very rare decays of the Higgs boson. The decay $H \rightarrow \phi \gamma$ is sensitive to the $H$ ss coupling. An ATLAS search for this decay using $\phi \rightarrow$ $K^{+} K^{-}$has set an upper limit of $\mathscr{B}_{H \rightarrow \phi \gamma}<1.4 \times 10^{-3}$ [18], a few orders of magnitude larger than the SM prediction of $2.3 \times 10^{-6}$. Additionally, rare non-SM decays can probe new Higgs-related couplings, such as the lepton-flavour-violating decays $H \rightarrow e \mu, H \rightarrow e \tau$, and $H \rightarrow \mu \tau$, and a search for the latter from CMS in Run 1 had an excess above background with a significance of $2.4 \sigma$. An update of this search in Run 2 does not confirm the excess and sets a limit of $\mathscr{B}_{H \rightarrow \mu \tau}<0.012$ [19].

Higgs-boson decays can also be affected by additional scalars and an extended Higgs sector. An additional scalar singlet $a$ with a low mass would allow $H \rightarrow a a$ decays through scalar mixing. The singlet could then decay to fermions through this mixing. Searches for such decays at CMS have provided limits on the $H \rightarrow a a$ branching fraction using $a \rightarrow b b, \mu \mu$, and $\tau \tau$ decays [20]. Searches for new scalars at high mass have resulted in limits on: a neutral scalar $X$ decaying to $\gamma \gamma$ [21], $Z \gamma$ [22], $Z Z$ [23], $W W$ [24], and $\tau \tau$ [25]; a singly-charged Higgs boson in $H^{ \pm} \rightarrow \tau \nu$ [26] and $H^{ \pm} \rightarrow t b$ [27]; and a doubly-charged Higgs boson in $H^{ \pm \pm} \rightarrow e e$ [28]. Indirect sensitivity to $H^{ \pm \pm}$is possible through lepton-flavour-violating decays, and the most sensitive limit on $\tau \rightarrow 3 \mu$ from Belle sets a lower limit on the mass of $H^{ \pm \pm}$of $\approx 2 \mathrm{TeV}$ for a neutrino mass scale of $0.1 \mathrm{eV}$ in the context of the Type-II Seesaw Model. Recent searches from ATLAS and LHCb suggest that the LHC will improve these constraints, while Belle II and a potential future circular $e^{+} e^{-}$collider on the $Z$ resonance could extend the mass limits to $5 \mathrm{TeV}$ and $15 \mathrm{TeV}$, respectively [29]. 


\section{Resonance and general searches}

The production of a resonance in fermion-fermion scattering would provide the most direct sign of new physics. Resonance searches at the LHC probe for new particles decaying to lepton pairs, quark or gluon pairs, or pairs of vector or Higgs bosons. A prominent set of fermion searches test for the presence of new quarks with vector couplings to the weak gauge bosons ("vector-like quarks"). A number of non-LHC experiments also search for new particles, such as $\mathscr{O}(300 \mathrm{MeV})$ neutrinos or heavy states that can be modelled with contact interactions.

Searches for resonances decaying to a pair of leptons have historically provided discoveries of the weak gauge bosons and the heavy-flavour quarks. Since these discoveries, the sensitivity to such resonances has seen a fifty-fold increase. An ATLAS search in the $e e$ and $\mu \mu$ final states using the full 2015-2016 data set gives a lower mass limit of $4.5 \mathrm{TeV}$ on a new vector boson with fermion couplings equal to those of the $Z$ boson [30]. Indirect constraints from HERA on the effective qqee coupling give similar lower limits on new vector bosons with left-handed couplings to fermions [31]. In the more challenging decay to the $\tau \tau$ final state, CMS has set a lower mass limit of $2.1 \mathrm{TeV}$ for a new vector boson with Z-like couplings [32]. Such a search is however most relevant for new bosons with non-universal lepton-flavour couplings. When interpreting the data in these searches, it can be important to consider the photon-induced contribution to the dilepton mass spectrum. This contribution is a small effect for narrow resonances but can be non-negligible for broad resonances [33], and the LHC searches take this into account.

Resonances with strong coupling to quarks and gluons can probe the highest masses. Searches of the dijet mass distribution with the full 2015-2016 data set produces lower mass limits of $6 \mathrm{TeV}$ on excited quarks from both ATLAS [34] and CMS [35], and 8.9 TeV on quantum black holes from ATLAS. The latter is the highest limit of a directly produced state in LHC collisions. New particles could couple weakly to quarks and gluons, making searches at low mass important. Such searches are extremely challenging due to the large background, but CMS has produced a result using highly boosted resonances to suppress the background [36]. The limits are the strongest on the coupling to quarks of a new state in the mass range 100-300 GeV. Figure 3 shows the ATLAS dijet mass (left) and the CMS boosted jet (right) distributions.

Boosted vector and Higgs bosons are typically used as a tool to search for new high-mass vector bosons or gravitons decaying to these particles. Previous searches by ATLAS have shown excesses in $V H$ final states, and a $W H$ search with the full data set has a local $3.3 \sigma$ excess at an invariant mass of 3.0 TeV [37], as can be seen in Figure 4 (left). This excess is however not confirmed by a similar CMS search [38], shown in Figure 4 (right).

There are tight constraints on new fermions with SM couplings from precision electroweak measurements and Higgs boson production. New fermions with weak vector couplings are however possible, and a dedicated ATLAS search for pairs of vector-like "top" quarks have excluded such quarks with masses below $1.2 \mathrm{TeV}$ if they decay exclusively to $\mathrm{Zt}$ and $\mathrm{Ht}$ [39]. Allowing also decays to $W b$, vector-like top quark masses below $800 \mathrm{GeV}$ are excluded [40]. A CMS search for singly-produced vector-like top quarks decaying to $Z t$ excludes masses up to $1.4 \mathrm{TeV}$, depending on the couplings [41]. CMS also searches for pair-production of charge-5/3 vector-like quarks, setting a lower mass limit of $1.2 \mathrm{TeV}$ [42].

A general search for new neutrinos at NA62 uses the missing mass in $K \rightarrow \mu N$ decays, where 

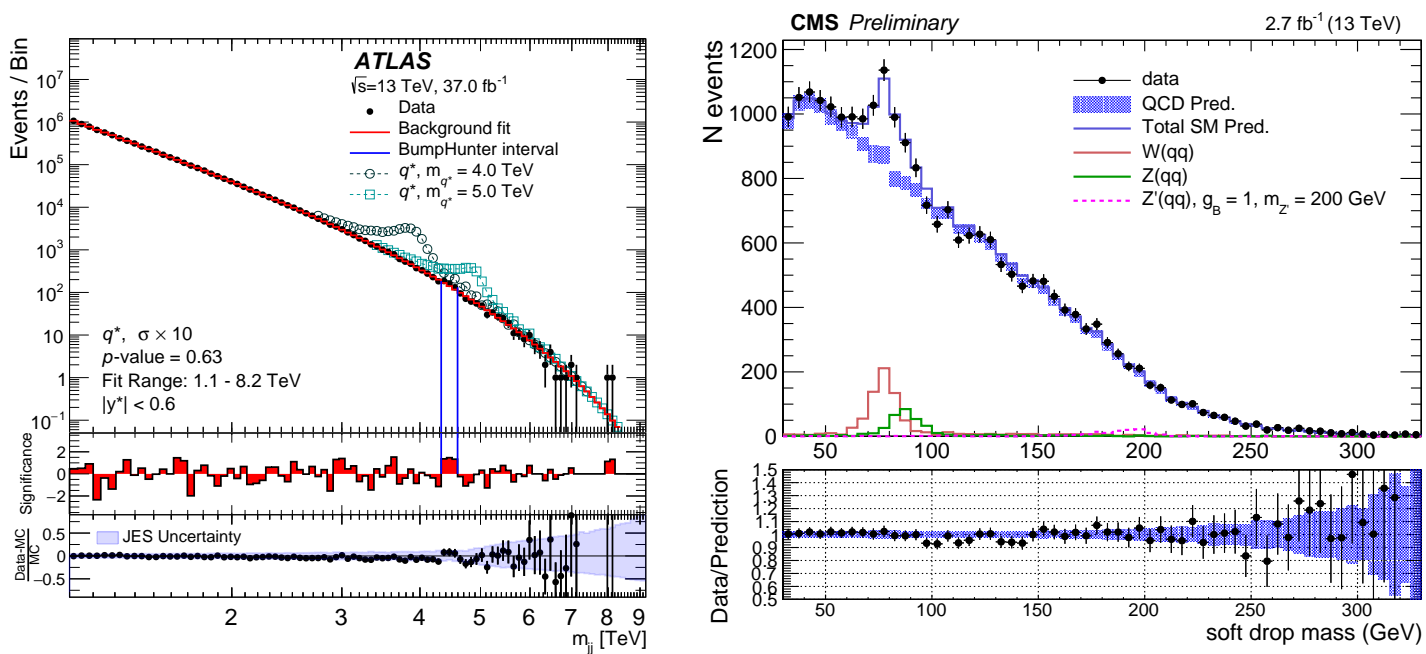

Figure 3: Left: The dijet mass distribution of an ATLAS search for new resonances [34]. Right: The invariant mass of the jet in a CMS search for boosted resonances [36].
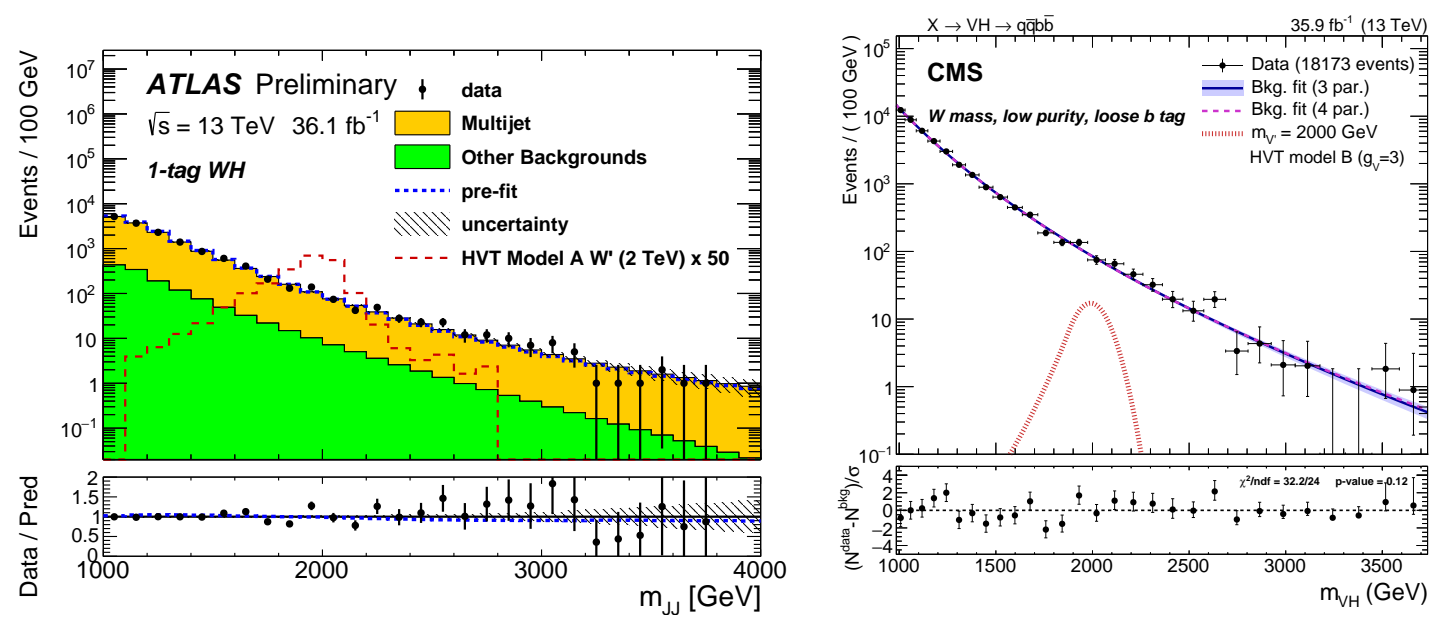

Figure 4: The invariant mass distribution of ATLAS [37] (left) and CMS [38] (right) searches for new resonances decaying to boosted $W$ and Higgs bosons. The ATLAS data has a $3.3 \sigma$ excess above background at a mass of $3.0 \mathrm{TeV}$ when including events with two identified $b$ quarks in the boosted jet associated with the Higgs boson. No excess is visible in the CMS data at this invariant mass.

the missing mass is $m=\sqrt{\left(p_{K}-p_{\mu}\right)^{2}}$ and $N$ is the new neutrino. The search constrains the square of the mixing strength between $N$ and the muon neutrino $\left(\left|U_{\mu 4}\right|^{2}\right)$ to be less than $\approx 10^{-5}$ for a neutrino mass in the range 300-375 MeV [43]. The analysis is based on 2007 data, while the 2015 data set is expected to provide sensitivity of a few times $10^{-8}$ on $\left|U_{\mu 4}\right|^{2}$.

\section{Dark matter and supersymmetry}

A typical experimental feature of searches for dark matter is an observed momentum imbal- 

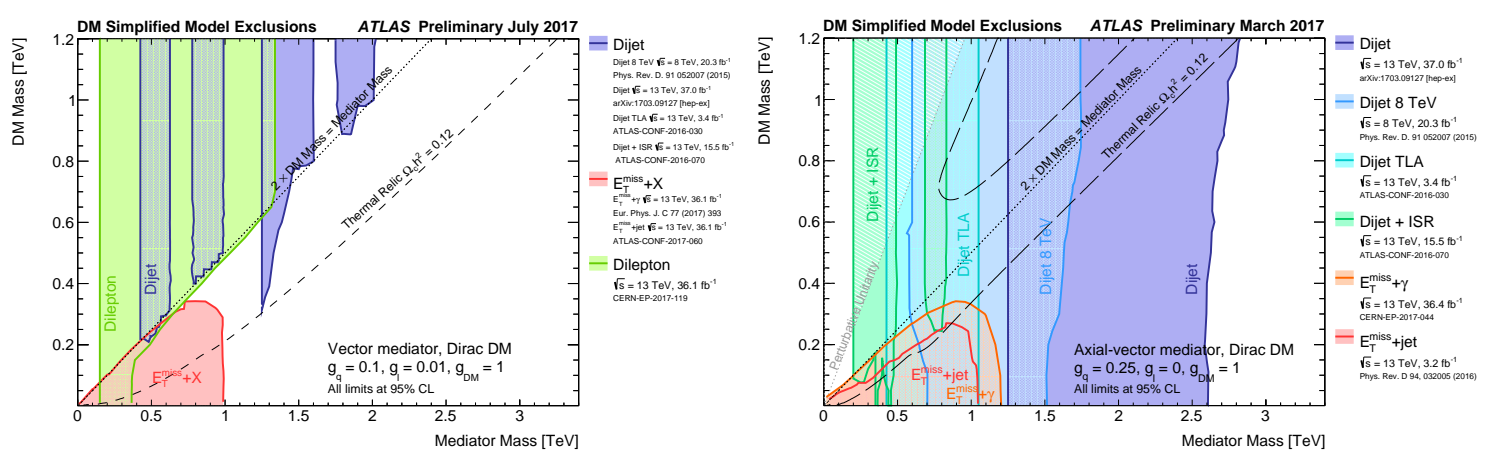

Figure 5: ATLAS constraints on the mass of the dark matter particle as a function of the mass of the mediator between fermions and dark matter, for a vector (left) and axial-vector (right) mediator. The couplings of the vector (axial-vector) mediator to dark matter, quarks, and leptons are $g_{D M}=1(1), g_{q}=0.1(0.25)$, and $g_{\ell}=0.01(0)$, respectively.

ance, from which the presence of the dark matter particle is inferred. Supersymmetric models with a dark-matter candidate share this feature, though supersymmetry searches also probe models without such a candidate. Indirect constraints on dark matter can also be obtained without the requirement of momentum imbalance. The recent increase in LHC $p p$ collision energy to $\sqrt{s}=13 \mathrm{TeV}$ has substantially extended the sensitivity of both categories of dark-matter and supersymmetry searches.

The most sensitive direct search for dark matter at the LHC uses high-momentum parton radiation to balance the momentum of the dark matter particle. In simplified models with a mediator between the quarks and the dark matter particles, dijet data extend the sensitivity to higher darkmatter and mediator mass. A summary of ATLAS limits based on these searches and those using dileptons or a radiated photon instead of a parton is shown in Figure 5. Not included in the figure is a recent ATLAS search for dark matter produced in association with a Higgs boson, where the Higgs boson decays to a $b$-quark pair [44]. Similar direct limits are obtained by the CMS experiment. Other searches assume a preferred mediator coupling to the third generation and search for dark matter in association with $t \bar{t}, t$, or $b \bar{b}$ [45]. Such searches are sensitive to the presence of vector-like quarks coupling to dark matter, with the sensitivity depending on the width of the vector-like quark [46].

The largest cross sections of supersymmetric models involve the production of squarks or gluinos, which subsequently decay to quarks, gluons, and (in models conserving R-parity) the lightest supersymmetric particle (LSP), which is a dark matter candidate. Searches for the pairproduction of gluinos probe a wide variety of possible decays, including $\tilde{g} \rightarrow b \bar{b} \tilde{\chi}^{0}, \tilde{g} \rightarrow t \bar{t} \tilde{\chi}^{0}$, $\tilde{g} \rightarrow q \bar{q} \tilde{\chi}^{0}$, and $\tilde{g} \rightarrow t \bar{b} \tilde{\chi}^{ \pm} \rightarrow t \bar{b} W \chi^{0}[47,48]$. Absent evidence for gluino production, limits on the gluino mass now approach $2 \mathrm{TeV}$ (Figure 6).

In general supersymmetric models there is a mass hierarchy between supersymmetric particles with and without strong couplings. Searches for charginos and neutralinos at lower mass will become more promising as the kinematic limits of the LHC are reached in gluino searches. Limits on the weakly-coupled supersymmetric particles are steadily increasing and now extend above 

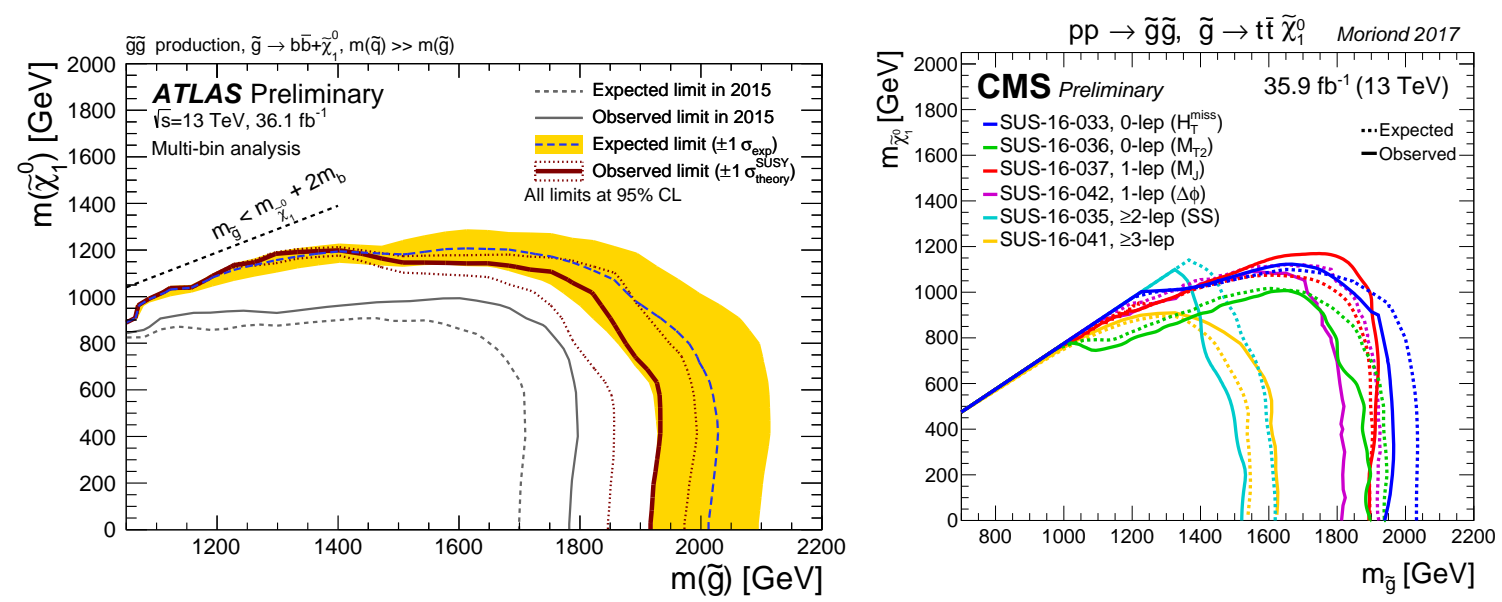

Figure 6: Constraints in the plane of gluino mass and LSP mass, for an ATLAS searches for gluino decays to $b \bar{b} \tilde{\chi}^{0}$ [48] (left) and CMS searches for gluino decays to $t \bar{t} \tilde{\chi}^{0}$ (right).
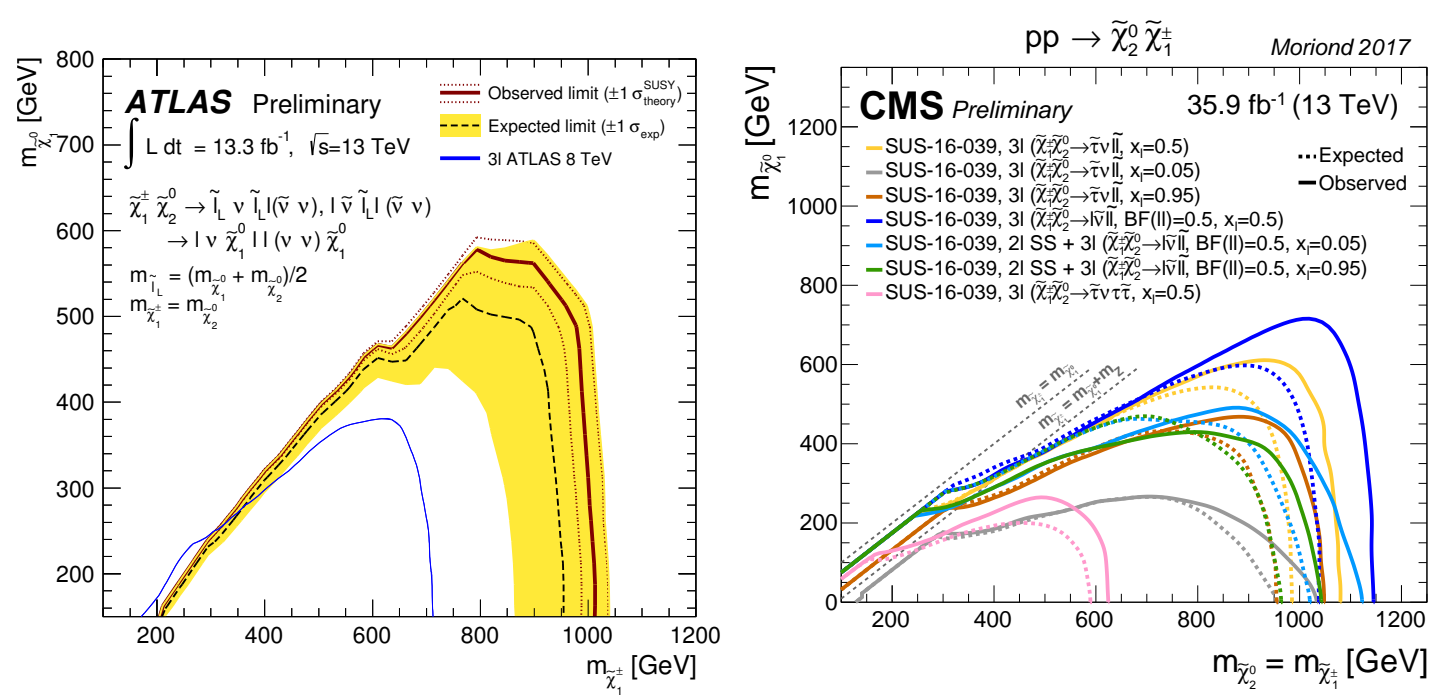

Figure 7: Constraints in the plane of chargino mass and LSP mass, for an ATLAS search for charginoneutralino production with decays through sleptons and a final state of three leptons and missing transverse momentum [50] (left), and for a variety of CMS searches (right).

$1 \mathrm{TeV}[49,50]$, as shown in Figure 7.

Aside from the presence of a dark-matter candidate, the strongest motivation for supersymmetry at the electroweak scale is its removal of the large fine-tuning associated with corrections to the Higgs boson mass. The correction from the top-quark loop is unbounded in the Standard Model and would drive the Higgs boson mass to the Planck scale in the absence of new physics. To minimize fine-tuning, the stop-quark mass should be similar to that of the top quark. Direct searches for stop-quark pairs now suggest some degree of tuning, as limits on the stop-quark mass are approaching $1 \mathrm{TeV}$ [51] (Figure 8). 

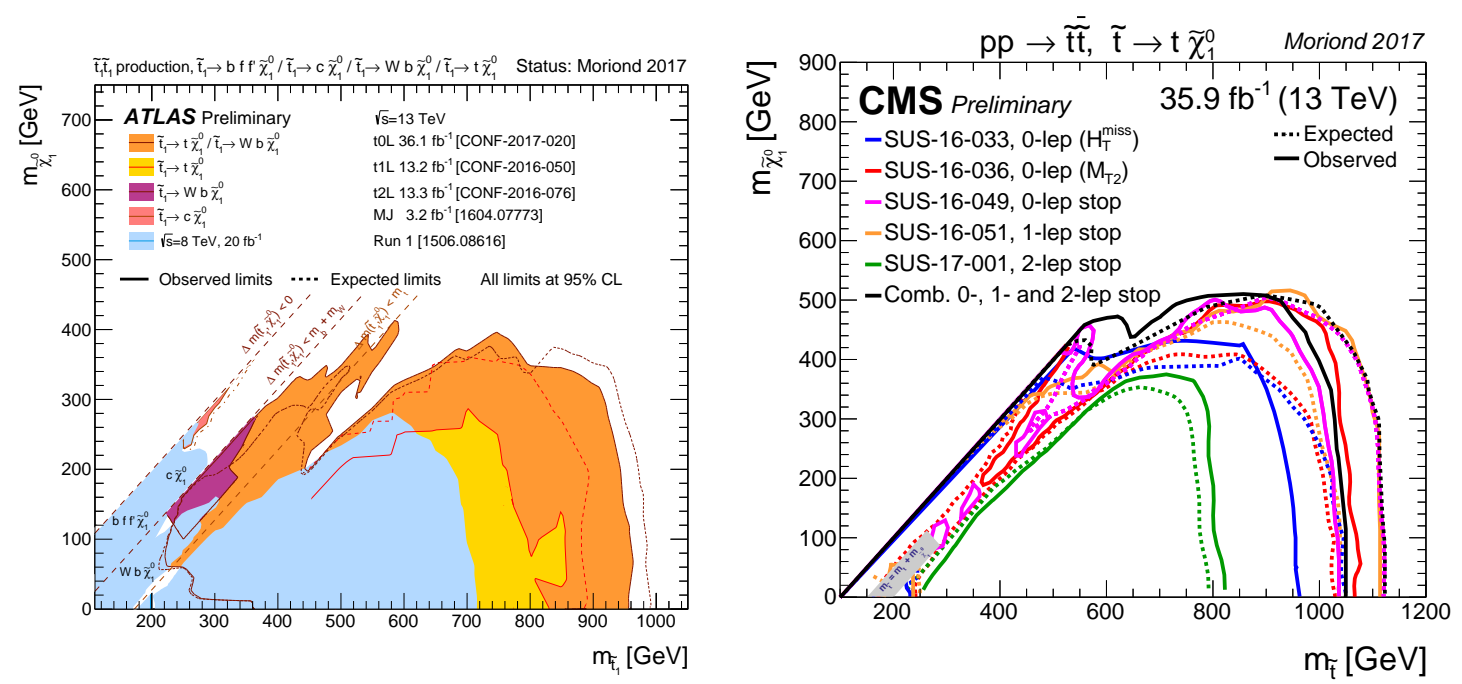

Figure 8: Constraints in the plane of stop-quark mass and LSP mass for a variety of ATLAS searches (left) and for CMS searches with the stop-quark decaying to a top quark and the LSP (right).

One way to avoid fine-tuning is to remove R-parity conservation from supersymmetry, at the cost of the dark matter candidate in the model. Searches for R-parity violating supersymmetric interactions are now extending into the $\mathrm{TeV}$ range [52], though the wide range of possible couplings allows unusual signatures such as displaced or disappearing tracks. A dedicated search for disappearing tracks excludes gluino masses in the TeV range [53].

Another way around fine-tuning is to add symmetries and additional particles at the electroweak scale. The simple addition of a $\mathrm{U}(1)$ gauge group with $B-L$ charge in supersymmetry removes the fine-tuning naturally, while keeping the dark matter candidate and providing a lowmass neutrino through the seesaw mechanism [54]. In models with an additional U(1) symmetry the $Z^{\prime}$ boson can have large branching fractions to non-SM particles, motivating dedicated searches at the LHC [55].

\section{Summary}

The first round of $\sqrt{s}=13 \mathrm{TeV}$ results have been produced by ATLAS and CMS, with no apparent evidence of new physics. Future collisions will substantially increase the size of the data set, though not the collision energy, so more work will be required to dig possible signals out of the data. The unobserved Higgs boson couplings to $b \bar{b}$ and $t \bar{t}$ will soon be measured, and rarer Higgs processes are not far away. Novel searches, particularly in unexpected signatures and unusual experiments, could still lead to a discovery in the near future. There is still plenty to explore at the electroweak scale!

\section{References}

[1] ATLAS Collaboration, ATLAS-CONF-2016-079 (2016). 
[2] CMS Collaboration, CMS-PAS-HIG-16-041 (2016). These results are from the version dated March 21, 2017.

[3] ATLAS Collaboration, ATLAS-CONF-2016-067 (2016).

[4] CMS Collaboration, CMS-PAS-HIG-17-015 (2017).

[5] N. Greiner, S. Höche, G. Luisoni, M. Schönherr, and J.-C. Winter, JHEP 01, 091 (2017) [arXiv:1608.01195].

[6] ATLAS Collaboration, ATLAS-CONF-2016-091 (2016).

[7] ATLAS Collaboration, ATLAS-CONF-2017-014 (2017).

[8] ATLAS Collaboration, ATLAS-CONF-2016-068 (2016).

[9] CMS Collaboration, CMS-PAS-HIG-17-004 (2017).

[10] A. Kulesza, L. Motyka, T. Stebel, and V. Theeuwes, arXiv:1704.03363 (2017).

[11] T. Jezo, J. Lindert, N. Moretti, and S. Pozzorini, these proceedings.

[12] C. Degrande, B. Fuks, K. Matawari, K. Mimasu, and V. Sanz, Eur. Phys. J. C77, 262 (2017) [arXiv:1609.04833].

[13] S. Boselli, C. M. Carloni Calame, G. Montagna, O. Nicrosini, F. Piccinini, and A. Shivaji, arXiv:1703.06667 (2017).

[14] J. Davies, M. Steinhauser, and D. Wellmann, Nucl. Phys. B920, 20 (2017) [arXiv:1703.02988].

[15] G. Heinrich, S. P. Jones, M. Kerner, G. Luisoni, and E. Vryonidou, arXiv:1703.09252 (2017).

[16] F. Bishara, R. Contino, and J. Rojo, Eur. Phys. J. C77, 481 (2017) [arXiv:1611.03860].

[17] CMS Collaboration, CMS-PAS-HIG-17-002 (2017).

[18] ATLAS Collaboration, Phys. Rev. Lett. 117, 111802 (2016) [arXiv:1607.03400].

[19] CMS Collaboration, CMS-PAS-HIG-16-005 (2016).

[20] CMS Collaboration, arXiv:1701.02032 (2017).

[21] CMS Collaboration, Phys. Lett. B767, 147 (2017) [arXiv:1609.02507].

[22] CMS Collaboration, CMS-PAS-EXO-16-034 (2016); CMS-PAS-EXO-16-035 (2016).

[23] CMS Collaboration, CMS-PAS-HIG-16-033 (2016); CMS-PAS-HIG-16-034 (2016); ATLAS Collaboration, ATLAS-CONF-2016-056 (2016); ATLAS-CONF-2016-079 (2016).

[24] CMS Collaboration, CMS-PAS-HIG-16-023 (2016); ATLAS Collaboration, ATLAS-CONF-2016-074 (2016).

[25] ATLAS Collaboration, ATLAS-CONF-2016-085 (2016).

[26] ATLAS Collaboration, ATLAS-CONF-2016-088 (2016).

[27] ATLAS Collaboration, ATLAS-CONF-2016-089 (2016).

[28] ATLAS Collaboration, ATLAS-CONF-2016-051 (2016).

[29] C. Hays, M. Mitra, M. Spannowksy, and P. Waite, JHEP 05, 014 (2017) [arXiv:1703.04362].

[30] ATLAS Collaboration, ATLAS-CONF-2017-027 (2017).

[31] O. Turkot, these proceedings. 
[32] CMS Collaboration, JHEP 02, 048 (2017) [arXiv:1611.06594].

[33] E. Accomando, J. Fiaschi, F. Hautmann, S. Moretti, and C.H. Shepherd-Themistocleous, Phys. Rev. D 95, 035014 (2017) [arXiv:1612.08168].

[34] ATLAS Collaboration, arXiv:1703.09127 (2017).

[35] CMS Collaboration, CMS-PAS-EXO-16-056 (2016).

[36] CMS Collaboration, CMS-PAS-EXO-16-030 (2016).

[37] ATLAS Collaboration, ATLAS-CONF-2017-018 (2017).

[38] CMS Collaboration, arXiv:1707.01303 (2017).

[39] ATLAS Collaboration, ATLAS-CONF-2017-015 (2017).

[40] ATLAS Collaboration, ATLAS-CONF-2016-104 (2016).

[41] CMS Collaboration, CMS-PAS-B2G-17-007 (2017).

[42] CMS Collaboration, CMS-PAS-B2G-16-019 (2016).

[43] C. Parkinson, these proceedings.

[44] ATLAS Collaboration, ATLAS-CONF-2017-028 (2017).

[45] CMS Collaboration, CMS-PAS-EXO-16-005 (2016); CMS-PAS-EXO-16-040 (2016); ATLAS Collaboration, ATLAS-CONF-2016-050 (2016); ATLAS-CONF-2016-076 (2016); ATLAS-CONF-2016-077 (2016); ATLAS-CONF-2016-086 (2016).

[46] S. Moretti, D. O’Brien, L. Panizzi, and H. Prager, Phys. Rev. D96, 035033 (2017).

[47] CMS Collaboration, CMS-PAS-SUS-16-033 (2016); CMS-PAS-SUS-16-036 (2016); CMS-PAS-SUS-16-037 (2016); CMS-PAS-SUS-16-042 (2016); ATLAS Collaboration, ATLAS-CONF-2017-022 (2017).

[48] ATLAS Collaboration, ATLAS-CONF-2017-021 (2017).

[49] ATLAS Collaboration, ATLAS-CONF-2016-075 (2016); ATLAS-CONF-2016-093 (2016); CMS Collaboration, CMS-PAS-SUS-16-039 (2016); CMS-PAS-SUS-16-043 (2016);

CMS-PAS-SUS-16-044 (2016); CMS-PAS-SUS-16-046 (2016); CMS-PAS-SUS-16-048 (2016).

[50] ATLAS Collaboration, ATLAS-CONF-2016-096 (2016).

[51] ATLAS Collaboration, ATLAS-CONF-2016-050 (2016); ATLAS-CONF-2016-076 (2016); ATLAS-CONF-2017-019 (2017); ATLAS-CONF-2016-020 (2017); CMS Collaboration, CMS-PAS-SUS-16-049 (2016); CMS-PAS-SUS-16-051 (2016); CMS-PAS-SUS-17-001 (2017).

[52] ATLAS Collaboration, ATLAS-CONF-2017-013 (2017).

[53] ATLAS Collaboration, ATLAS-CONF-2017-017 (2017).

[54] L. D. Rose, S. Khalil, S. J. D. King, C. Marzo, S. Moretti, and C. S. Un, arXiv:1702.01808 (2017).

[55] G. Corcella and S. Gentile, Eur. Phys. J. C75, 264 (2015). 